\title{
Intraoperative stereotactic injection of Indigo Carmine dye to mark ill-defined tumor margins: a prospective Phase I-II study
}

\author{
Konstantinos Margetis, MD, ${ }^{1}$ Prajwal Rajappa, MD, ${ }^{1}$ Apostolos John Tsiouris, MD, ${ }^{2}$ \\ Jeffrey P. Greenfield, MD, PhD, ${ }^{1}$ and Theodore H. Schwartz, MD, ${ }^{1,3-5}$ \\ Departments of ${ }^{1}$ Neurological Surgery, ${ }^{2}$ Radiology, ${ }^{3}$ Otorhinolaryngology, and ${ }^{4}$ Neurology, Brain and Spine Center; and \\ ${ }^{5}$ Department of Neuroscience, Brain and Mind Institute, Weill Cornell Medical College, NewYork-Presbyterian Hospital, New \\ York, New York
}

OBJECT A critical goal in neurosurgical oncology is maximizing the extent of tumor resection while minimizing the risk to normal white matter tracts. Frameless stereotaxy and white matter mapping are indispensable tools in this effort, but deep tumor margins may not be accurately defined because of the "brain shift" at the end of the operation. The authors investigated the safety and efficacy of a technique for marking the deep margins of intraaxial tumors with stereotactic injection of Indigo Carmine dye.

METHODS Investigational New Drug study approval for a prospective study in adult patients with gliomas was obtained from the FDA (Investigational New Drug no. 112680). At surgery, 1-3 stereotactic injections of $0.01 \mathrm{ml}$ of Indigo Carmine dye were performed through the initial bur holes into the deep tumor margins before elevation of the bone flap. White light microscopic resection was conducted in standard fashion by using frameless stereotactic navigation until the injected margins were identified. The resection of the injected tumor margins and the extent of resection of the whole tumor volume were determined by using postoperative volumetric MRI.

RESULTS In total 17 injections were performed in 10 enrolled patients (6 male, 4 female), whose mean age was 49 years. For all patients, the injection points were identified intraoperatively and tumor was resected at these points. The staining pattern was reproducible; it was a sphere of stained tissue approximately $5 \mathrm{~mm}$ in diameter. A halo of stained tissue and a backflow of dye through the needle tract were also noted, but these were clearly distinct from the staining pattern of the injection point, which was vividly colored and demarcated. Postoperative MR images verified the resection of all injection points. The mean extent of resection of the tumor as a whole was $97.1 \%$. For 1 patient, a brain abscess developed on postoperative Day 16 and needed additional surgical treatment.

CONCLUSIONS Stereotactic injection of Indigo Carmine dye can be used to demarcate multiple deep tumor margins, which can be readily identified intraoperatively by using standard white light microscopy. This technique may enhance the accuracy of frameless stereotactic navigation and increase the extent of resection of intraaxial tumors.

Clinical trial registration no.: NCT01767415 (clinicaltrials.gov)

http://thejns.org/doi/abs/10.3171/2014.9.JNS14113

KEY WORDS extent of resection; glioma; Indigo Carmine; frameless stereotaxy; neuronavigation; oncology

$\mathrm{F}$ OR maximizing overall and recurrence-free survival in patients with low- and high-grade gliomas, the extent of resection is critical. ${ }^{9,10}$ However, the margins between neoplastic tissue and normal parenchyma are often difficult to distinguish intraoperatively by macroscopic or microscopic visualization. The optical properties of neoplastic tissue and normal parenchyma can be similar.
The transition from neoplastic to normal tissue can also be poorly demarcated, with indistinct transitions between these tissue types at the microscopic level. A major advance in the surgical treatment of these intraaxial pathologies was the use of preoperative MRI as an intraoperative aid in the form of stereotactic guidance. However, "brain shift" caused by loss of CSF, cyst decompression, tumor

ABBREVIATIONS 5-ALA = 5-aminolevulinic acid; ETL = echo-train length; IND = Investigational New Drug; NEX = number of excitations.

SUBMITTED January 16, 2014. ACCEPTED September 25, 2014.

INCLUDE WHEN CITING Published online October 31, 2014; DOI: 10.3171/2014.9.JNS14113.

DISCLOSURE The study was supported by the Department of Neurological Surgery of Weill Cornell Medical College. The authors have no personal, financial, or institutional interest in any of the drugs, materials, or devices described in this article. 
removal, edema, and/or gravity can contribute to inaccuracies in frameless navigation, particularly at deep margins; error can be as much as $3 \mathrm{~cm} .{ }^{12}$ Intraoperative MRI presents a potential solution, but the cost is often prohibitive. ${ }^{20}$ Tumor-specific dyes are not readily available in the United States, and the interpretation and significance of vague fluorescence can be ambiguous without sophisticated quantitative measurements (http://www.cancer.gov/ clinicaltrials/search/view?cdrid $=674659 \&$ version $=$ Health Professional).

Another solution to the problem is marking the margins of the tumor by using stereotactic navigation at the beginning of the operation, before the brain has shifted. This concept has been realized with the placement of catheters as "fence posts" around the margin of the tumor.,15 This technique has certain limitations: the catheters must be placed after a craniotomy has been performed, so some degree of shift has already occurred; the catheters can be dislodged; and the catheters are most useful for marking the perimeter of the tumor and not the deep margin. To overcome these limitations, we developed a new technique involving the stereotactic injection of indigotindisulfonate sodium (Indigo Carmine, Akorn, Inc.) into the deep tumor margins through small bur holes before opening the craniotomy and dura. Indigo Carmine is widely used in urological surgery to identify the ureteral orifices after intravenous administration of the dye. This dye has also been used within the CNS, administered intrathecally or intraventricularly, for CSF flow studies. ${ }^{16}$ Hence, its safety has been studied. Our concept was to inject a small volume of dye to mark the deep tumor margins by using a stereotactically guided needle passed through a bur hole in the dura. After injection of the dye into as many locations as needed, the surgery could proceed in a standard fashion and the injected margin would always be labeled regardless of brain shift. In this study, we investigated the safety, feasibility, and preliminary efficacy of the stereotactic injection of Indigo Carmine for the marking of illdefined brain tumor margins.

\section{Methods \\ Approval for the Clinical Study}

The study was reviewed and approved by the Weill Cornel Medical School Institutional Review Board and by the FDA with an Investigational New Drug (IND) application (IND no. 112680). The study was registered with the ClinicalTrials.gov database (http://clinicaltrials.gov), and its registration number is NCT01767415. The study was conducted in accordance with the ethical principles that have their origins in the Declaration of Helsinki. Written informed consent for study participation was obtained from each patient at the same time and place that informed consent for the surgery was obtained.

\section{Cadaveric Study}

Before initiation of the study, a preliminary experiment was performed in which Indigo Carmine was injected into fixed cadaveric brains. The goal was to create a 5-mm sphere of stained tissue. Using a 20-gauge spinal needle and a tuberculin syringe, we injected different aliquots of
Indigo Carmine (0.01-0.5 ml) into the brains. Brains were sliced, and the area of dye spread was measured.

\section{Human Trial}

To study the safety, feasibility, and potential utility of the method, we designed an open, prospective Phase I-II study without control groups. The study enrolled patients with a presumed diagnosis of glioma, scheduled for resection. All patients with high- and low-grade gliomas were offered the opportunity to participate in the study; only those who agreed to participate were included. Exclusion criteria were pregnancy and age younger than 18 years. The study was conducted at NewYork-Presbyterian/Weill Cornell Medical College, from September 2012 through April 2014.

The participants underwent preoperative MRI, which was used for the frameless stereotaxy according to the current standard of care. The tumor margins that were thought to be the most difficult to achieve, according to assessment of the surgeon (T.H.S.), were targeted for injection. Demarcation of the tumor boundaries on the preoperative MR images was based on hyperintense T2/ FLAIR signal and/or pathological enhancement as described below. Eloquent areas were localized according to anatomical landmarks and/or specialized studies that were performed as indicated (Table 1). The criteria for selection of the injection points included areas that would be subjected to significant brain shift, deep areas, areas close to eloquent white matter tracts, or the depth of fingerlike projections of tumor tissue inside normal parenchyma that could be missed during tumor resection. All points were close to the tumor-brain margins and located inside the pathological tissue intended for resection.

After the skin flap was elevated, one to several bur holes were opened at the margins of the intended craniotomy flap and occasionally at the center of the flap. A 3 -sphere "star" was attached to the base of a 20-gauge spinal needle (Fig. 1). The spinal needle was then registered to the stereotactic system (Brainlab, Inc.). The stylet was removed, and a tuberculin syringe containing Indigo Carmine dye (5-ml ampules $/ 8 \mathrm{mg} / \mathrm{ml})$ was connected. A small amount of Indigo Carmine dye was flushed through the needle to eliminate any dead space until $0.01 \mathrm{ml}$ of dye remained in the syringe. Through one or more bur holes, the spinal needle was stereotactically passed through the dura into the brain, targeting the predetermined injection points. The selection of the bur holes through which to inject the Indigo Carmine dye was based on an attempt to keep the trajectory of the needle almost exclusively through pathological tissue or tissue intended for resection. We did not standardize the number of bur holes or the location of the injection site; this determination was made by the operating surgeon, who determined the margin of the tumor thought to be most challenging to appreciate during surgery. The trajectory did not cross blood vessels or sulci. When the tip of the spinal needle reached the predetermined point, the dye was infused and the needle was withdrawn. This procedure was repeated according to the number of predetermined injection points. After the infusions were completed, the surgery was performed. Other surgical adjuncts were also used according to the stan- 
TABLE 1. Clinical data for study participants

\begin{tabular}{|c|c|c|c|c|c|c|}
\hline $\begin{array}{l}\text { Patient } \\
\text { No. }\end{array}$ & $\begin{array}{l}\text { Age (yrs), } \\
\text { Sex }\end{array}$ & Clinical Presentation & Location & $\begin{array}{l}\text { Specialized Studies/Surgical } \\
\text { Adjuncts* }\end{array}$ & $\begin{array}{l}\text { LOS } \\
\text { (days)† }\end{array}$ & $\begin{array}{l}\text { Postop Clinical Course \& } \\
\text { Complications }\end{array}$ \\
\hline 1 & $46, M$ & Seizures & Lt temporal & $\begin{array}{l}\text { Wada test, fMRI (will not resect } \\
\text { hippocampus because of retained } \\
\text { memory there), awake craniotomy } \\
\text { for language mapping }\end{array}$ & 3 & $\begin{array}{l}\text { No postop seizures, mild tem- } \\
\text { porary difficulty w/ word } \\
\text { finding in immediate postop } \\
\text { period }\end{array}$ \\
\hline 2 & $74, \mathrm{M}$ & Incidental (fall) & Lt frontal & None & 9 & Good \\
\hline 4 & $63, \mathrm{~F}$ & Headache & Lt occipital & None & 3 & Good \\
\hline 5 & $25, \mathrm{~F}$ & Incidental (bicycle accident) & Rt parietal & None & 3 & Good \\
\hline 6 & $58, M$ & $\begin{array}{l}\text { Recurrent GBM w/ baseline It } \\
\text { hemiparesis }\end{array}$ & Rt frontal & $\begin{array}{l}\text { Brachytherapy, SSEP, cortical } \\
\text { stimulation }\end{array}$ & 14 & $\begin{array}{l}\text { Temporary increased It-sided } \\
\text { hemiparesis }\end{array}$ \\
\hline 9 & $61, M$ & $\begin{array}{l}\text { Neurological deficit (language } \\
\text { difficulties). Hx of ganglioma } \\
\text { resected } 7 \text { yr ago }\end{array}$ & Lt parietal & None & 2 & Good \\
\hline 10 & $27, \mathrm{~F}$ & $\begin{array}{l}\text { Recurrent oligoastrocytoma } \\
\text { (WHO Grade II) }\end{array}$ & Lt frontal & SSEP, cortical stimulation & 3 & Good \\
\hline
\end{tabular}

EVD = external ventricular drain; fMRI = functional MR imaging; GBM = glioblastoma multiforme; Hx = history; LOS = length of stay; RT = radiotherapy; SSEP = somatosensory evoked potential.

* Studies in addition to frameless stereotaxy.

$\dagger$ Length of stay after surgery.

dard of care, such as awake craniotomy and brain mapping. Frameless stereotaxy was used in all cases. Standard image-guided microscope-based surgery was performed to remove the tumor.

The tumor was resected either in its entirety or subtotally, depending on the goal of surgery. This goal was determined after appreciating the relationship of the tumor's margins with eloquent brain tissue and weighing the relative risks and benefits of maximizing the extent of resection. The operating surgeon made this determination after consulting with the patient and determining the patient's wishes by using the standard informed consent procedure. Dye was injected into areas intended for resection according to the aforementioned decision-making process.

Intraoperatively, the surgeon noted whether the dye

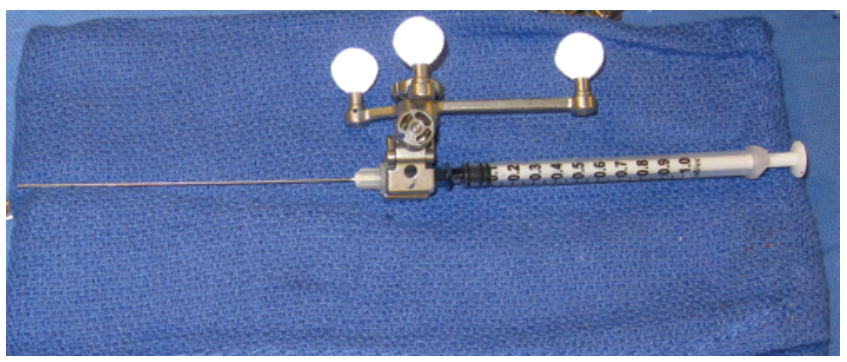

FIG. 1. The technical equipment required for the injection, provided a neuronavigation system is available, is minimal and readily available: a 20 -gauge spinal needle and a tuberculin syringe. An attachment for the registration of instruments with the stereotactic system (a "star") is connected to the spinal needle. Figure is available in color online only. was observed and how far the stained tissue had moved from the predetermined area, according to intraoperative stereotactic navigation, because of the expected shift of the brain during surgery. For evaluation of the extent of resection and investigation of the resection of tumor at the injection points, postoperative brain MRI was performed, according to the standard of care, for all patients.

The feasibility of this method was evaluated by assessing the visibility and the diffusion properties of Indigo Carmine in the tumor tissue. The efficacy of this method was assessed according to the study hypothesis that if the stained tissue was recognized and resected intraoperatively, then no residual tumor would be seen at these sites on the postoperative MR images. We also assessed the extent of tumor resection on the postoperative MR images. Volumetric analysis was performed by a board-certified neuroradiologist with 10 years of experience (A.J.T.) on a General Electric Advantage workstation (version 4.3). For the volumetric analysis, a volume viewer tool with autosegmentation capabilities was used. For the estimation of the enhancing tumor volume on preoperative and postoperative MR images, we used 1.5-mm post-Gd (Gadovist [1.0 $\mathrm{mmol} / \mathrm{ml}$, Bayer) 3D T1-weighted spoiled gradient recall echo or 5-mm post-Gd (Gadovist [1.0 $\mathrm{mmol} / \mathrm{ml}])$ axial T1weighted sequences, respectively. For the estimation of the T2 hyperintense tissue volume, we used 5-mm axial T2-weighted or T2-weighted FLAIR sequences. The MRI parameters for the 3D T1-weighted spoiled gradient recall echo were 1.5-mm slice thickness, no gap; TR $6 \mathrm{msec}$, TE $1.8 \mathrm{msec}$, matrix $256 \times 256$, number of excitations (NEX) 
1, echo-train length (ETL) 1, and flip angle $25^{\circ}$. The MRI parameters for the axial T1-weighted fast spin echo were 5-mm slice thickness, 0.5 gap; TR $533 \mathrm{msec}$, TE $14 \mathrm{msec}$, matrix $288 \times 192$, NEX 2, ETL 2, and flip angle $90^{\circ}$. The MRI parameters for the axial T2-weighted fast spine echo FLAIR were 5-mm slice thickness, 0.5 gap; TR 9000 msec, TE 142 msec, matrix $256 \times 192$, NEX 1, ETL 1, and flip angle $90^{\circ}$.

The deviation between the stereotactic location of the injection point and the stereotactic location where the injection point was located intraoperatively was noted if it was larger than $1 \mathrm{~cm}$. Safety was assessed by monitoring for any complications or adverse effects, even if they seemed to be unrelated to the Indigo Carmine infusion.

In Phase I of the study (first 5 patients), the Indigo Carmine injection did not guide clinical decision making. At the end of this phase, an evaluation was made on whether the intraoperative recognition and resection of stained tissue correlated with the resection of tumor at the injection points as determined by postoperative MRI. If the evaluation confirmed this correlation, the study proceeded to Phase II, in which Indigo Carmine was included with the other adjuncts used by the surgeon to guide resection.

For any patient, the study would be terminated if any unexpected neurological or cardiovascular abnormalities (e.g., seizures, pulse rate or blood pressure instability) developed during dye injection. The study as a whole would be terminated if adverse events developed in more than 3 patients and/or a satisfying staining pattern could not be achieved in more than 5 patients. Because of the development of a brain abscess in 1 patient over the course of the study, study termination criteria were expanded to include development of another brain abscess in any subsequently enrolled patient.

\section{Results \\ Cadaver Study}

Examination of the cadaveric experiments revealed that the ideal quantity of dye required to create a $5-\mathrm{mm}$ sphere of stained tissue was approximately $0.01 \mathrm{ml}$ (Fig. 2). These experiments also showed that injection into CSF spaces might result in significant diffusion of the dye into these spaces. Additionally, some backflow of the dye through the needle tract occurred. After these studies, it was determined that $0.01 \mathrm{ml}$ would be administered.

\section{Human Trial}

A total of 10 patients (6 male and 4 female) were enrolled in the study; their mean age was 49 years (Table 1). All patients had gliomas of varying grades (Table 2). Tumor diagnosis was an incidental finding in 3 patients; recurrent disease was diagnosed in 3 patients; and seizures were experienced by 1 patient, headache by 1 patient, and a neurological deficit by 2 patients. The tumors were located in the frontal, parietal, and occipital lobes on both sides. The median postoperative hospital stay was 3 days (range 2-14, mean 4.7 days). The mean follow-up time was 5.9 months (median 6.5, range 2-8 months).

The goal of the Indigo Carmine injection varied according to the imaging characteristics of the tumor. For low-

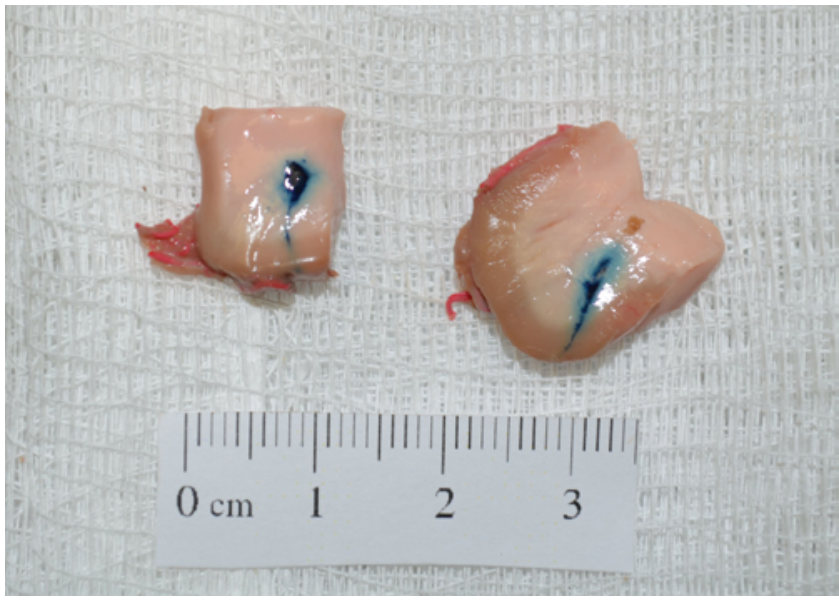

FIG. 2. Injection, with a tuberculin syringe, of approximately $0.01 \mathrm{ml}$ of Indigo Carmine into cadaveric brains produced a diffusion pattern that had a diameter of a few millimeters. Backflow through the needle tract toward the cortical entry point is also noted. Figure is available in color online only.

grade tumors, Indigo Carmine was injected at the deepest margin of T2 signal hyperintensity. For higher-grade and enhancing tumors, the injection was made at the deepest margin of the enhancing component of the tumor. For the 10 patients, 17 Indigo Carmine injections were made. The diffusion patterns of the dye were very satisfactory; in all patients, a distinct blue area could be identified in the area corresponding to the injection point (Video 1).

VIDEO 1. Clip showing 2 illustrative resections of tumor at the Indigo Carmine injection points. Copyright Theodore Schwartz. Published with permission. Click here to view with Media Player. Click here to view with Quicktime.

In some patients, a small degree of backflow through the needle tract could be identified (Fig. 3). In others, a halo of lightly colored tissue could be seen proximal to the injection point, probably the result of dye leakage as the needle was withdrawn. The halo was very faint, and in all patients it could be differentiated from the vividly colored injec-

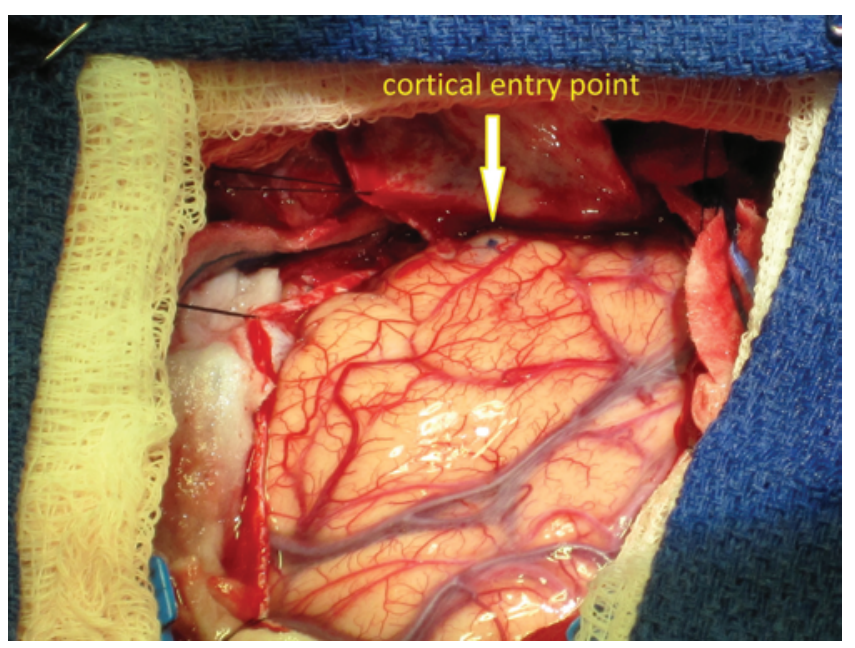

FIG. 3. Backflow through the needle tract onto the cortical surface was noted in some cases, which led to the marking of the cortical entry point with an approximately $2-\mathrm{mm}$-diameter blue-stained pattern. Figure is available in color online only. 
TABLE 2. Radiological and pathological parameters of study participants

\begin{tabular}{|c|c|c|c|c|c|c|}
\hline \multirow{2}{*}{$\begin{array}{l}\text { Patient } \\
\text { No. }\end{array}$} & \multirow{2}{*}{$\begin{array}{l}\text { No. of Dye } \\
\text { Injections }\end{array}$} & \multirow{2}{*}{$\begin{array}{l}\text { Intraop } \\
\text { Identification } \\
\& \text { Resection of } \\
\text { Injected Sites }\end{array}$} & \multirow[b]{2}{*}{ Pathology } & \multicolumn{3}{|c|}{ Tissue Intended for Resection } \\
\hline & & & & Preop Vol in $\mathrm{cm}^{3}$ & Postop Vol in $\mathrm{cm}^{3}$ & EOR in \% \\
\hline 1 & 2 & Yes & $\begin{array}{l}\text { Anaplastic astrocytoma } \\
\text { (WHO Grade III) }\end{array}$ & $\begin{array}{l}80.4 \text { (T2 hyperintense tis- } \\
\text { sue), } 0.5 \text { (enhancing tu- } \\
\text { mor) }\end{array}$ & $\begin{array}{l}7.25 \text { (T2 hyperintense tis- } \\
\text { sue), } 0 \text { (enhancing tumor) }\end{array}$ & $\begin{array}{l}91 \text { (T2 hyperintense } \\
\text { tissue), } 100 \text { (en- } \\
\text { hancing tumor) }\end{array}$ \\
\hline 4 & 3 & Yes & GBM & 8.3 (enhancing tumor) & 0 (enhancing tumor) & 100 (enhancing tumor) \\
\hline 5 & 1 & Yes & $\begin{array}{l}\text { Anaplastic astrocytoma } \\
\text { (WHO Grade III) }\end{array}$ & 12.6 (T2 hyperintense tissue) & 0 (T2 hyperintense tissue) & $\begin{array}{l}100 \text { (T2 hyperintense } \\
\text { tissue) }\end{array}$ \\
\hline 6 & 1 & Yes & GBM & 13.5 (enhancing tumor) & 0.4 (enhancing tumor) & 97 (enhancing tumor) \\
\hline 9 & 1 & Yes & $\begin{array}{l}\text { Anaplastic astrocytoma } \\
\text { (WHO Grade III) }\end{array}$ & 5.7 (T2 hyperintense tissue) & 0.4 (T2 hyperintense tissue) & $\begin{array}{l}93 \text { ( } \mathrm{T} 2 \text { hyperintense } \\
\text { tissue) }\end{array}$ \\
\hline 10 & 1 & Yes & $\begin{array}{l}\text { Oligoastrocytoma (WHO } \\
\text { Grade II) }\end{array}$ & 40.7 (enhancing tumor) & 0 (enhancing tumor) & 100 (enhancing tumor) \\
\hline
\end{tabular}

$\mathrm{EOR}=$ extent of resection.

tion point (Fig. 4). For all 17 injections, the injection points were identified and tumor was resected at these points. By comparing the captured images of the preoperative trajectory and injection site with the postoperative volumetric MR images, we evaluated the extent of tumor resection at the injection points. The postoperative MR images verified $100 \%$ resection at the points that were marked with Indigo Carmine, with clean tumor margins in these areas (Fig. 5). With regard to the whole tumor volume, the mean extent of resection of the tissue intended for resection was
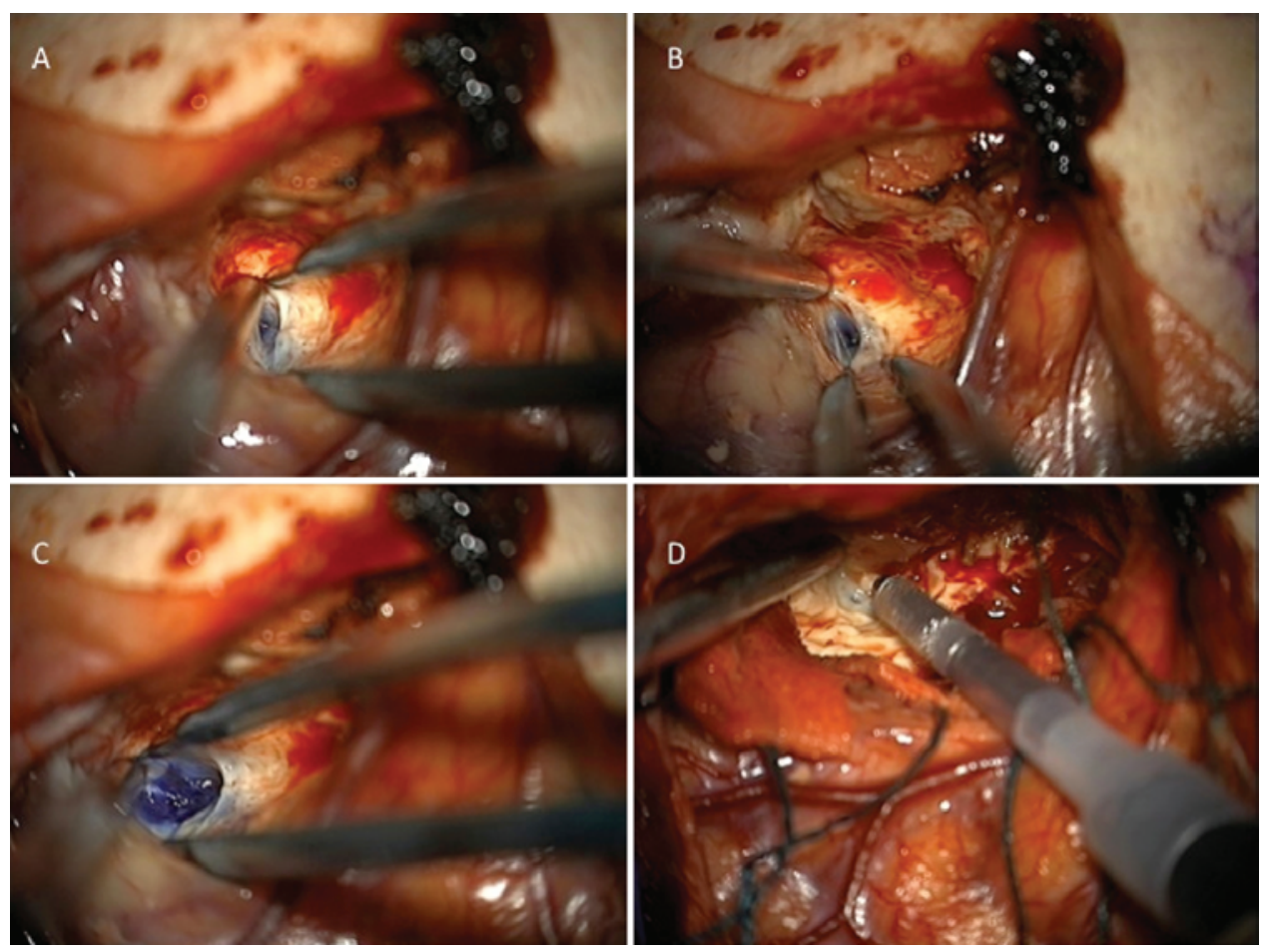

FIG. 4. Resection of an Indigo Carmine injection point. A and B: Initial recognition of the halo surrounding the injection point and identification of a small area of the vividly stained injection point. C and D: Entry of (C) and resection of (D) the vividly stained sphere of tissue that corresponds to the injection point. Figure is available in color online only. 

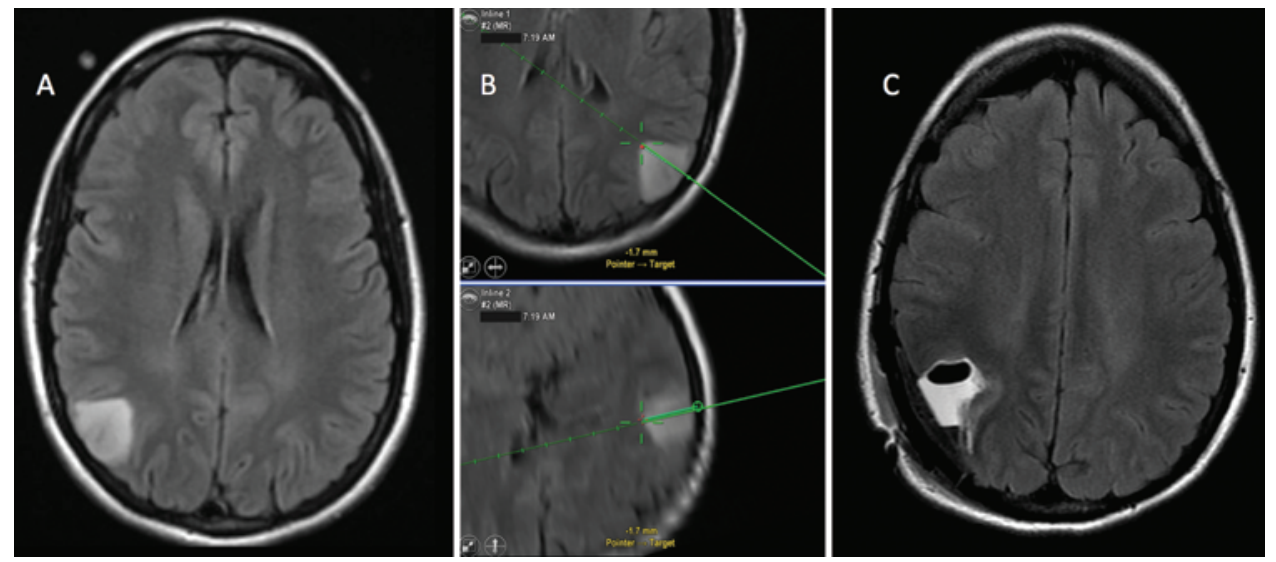

FIG. 5. Illustrative case of the use of Indigo Carmine. A: Preoperative FLAIR image of a nonenhancing lesion that is wedge shaped and cortical based. B: Screenshots from the neuronavigation screen showing the injection point of Indigo Carmine that corresponds to the deepest point of the lesion. C: Postoperative FLAIR image. After the intraoperative recognition of the bluestained injection point, the deepest point of the tumor was resected without inadvertent excessive entry into normal parenchyma. Figure is available in color online only.

97.1\% (SD 3.54\%). In Patient 8, the stereotactic location of the injection point deviated $15.4 \mathrm{~mm}$ from the stereotactic location where it was identified intraoperatively (Fig. 6). This deviation was attributed to either brain shift during to the development of the resection cavity and/or intraoperative mild edema. Had we relied solely on the stereotactic navigation, we would have resected normal brain tissue in that patient.

During the follow-up period, 1 patient died of disease progression 7 months after surgery. No complications occurred intraoperatively in any patient. Another patient experienced transient worsening of preexisting hemiparesis, which resolved. In another patient, postoperative deep Staphylococcus aureus infection (brain abscess in the resection cavity) developed on postoperative Day 16. Of note, in this patient the resection cavity was used as a path for intraoperative placement of an external ventricular drainage catheter. The adverse event was expeditiously reported to the Institutional Review Board and the FDA, and the study was temporarily placed on hold. The study protocol was modified by the addition of a study termination rule, which was the development of brain abscess in any subsequently enrolled patient. Investigations into the Indigo Carmine batch did not reveal any evidence of contamination, and the precise cause of the infection was never determined.

\section{Discussion}

The initial results of this study show that frameless stereotactic injection of Indigo Carmine through bur holes can be used to mark deep tumor margins that can be identified during surgery and adequately removed. Using bur holes enables the surgeon to make the injections before any brain shift, and these holes can then be used for the craniotomy. One limitation of this technique is that only a limited number of points can be injected, which is clearly not a complete representation of the complex 3D tumor margin. However, as most surgeons will attest, only a limited number of deep tumor margins cause the greatest concern for the surgeon, and the ability to reliably identify these margins during surgery conveys a significant advantage, both psychologically and literally. Although we cannot state from the current study that this method provides statistically significant benefit with respect to extent of resection or patient survival, the data provide proof of concept for marking deep tumor margins with an injected dye. Given the current literature on the association between extent of resection and overall and progression-free survival for patients with gliomas, one can extrapolate the benefit of any technique that safely increases extent of resection. ${ }^{9,10}$ In this study, the MRI was conducted according to the current standard of care; therefore, slice thicknesses differed in the postgadolinium preoperative and postoperative MRI studies. Although doing so might introduce a small degree of error, because of the different resolution of the imaging studies, we believe that it did not affect the validity of the results.

The overall safety profile seemed to be satisfactory but definitely warrants further study. For the 1 infectious complication, it cannot definitively be determined whether the adverse event was caused by the clinical procedure or the injection of the Indigo Carmine. During surgery, the stained area was identified and resected as was the whole tract of the needle, which was through tumor tissue. Of note, an external ventricular drain was placed at the end of this patient's procedure because the ventricular system was entered intraoperatively. A literature search for postoperative infections in glioblastoma multiforme patients identified 2 recent publications that report infection rates of $4.7 \%$ and $5.08 \% .^{1,2}$ The causative microorganisms of the infection were Staphylococcus spp. in 58\% and $70 \%$ and specifically S. aureus in $23.5 \%$ and $60.0 \%$ in each of the 2 studies. Thus far in our study, Indigo Carmine was injected in 10 patients, and 1 infectious complication occurred. Fisher exact test results suggest that there is no statistically significant difference between the infection rate observed in the study up to this time (1 in 10) and the reported postoperative infection rate of 5\% among patients with glioblastoma multiforme $(\mathrm{p}=0.4434)$.

Use of Indigo Carmine in the CNS was first reported in 1923. ${ }^{11}$ Although it was used extensively in the pre-CT 

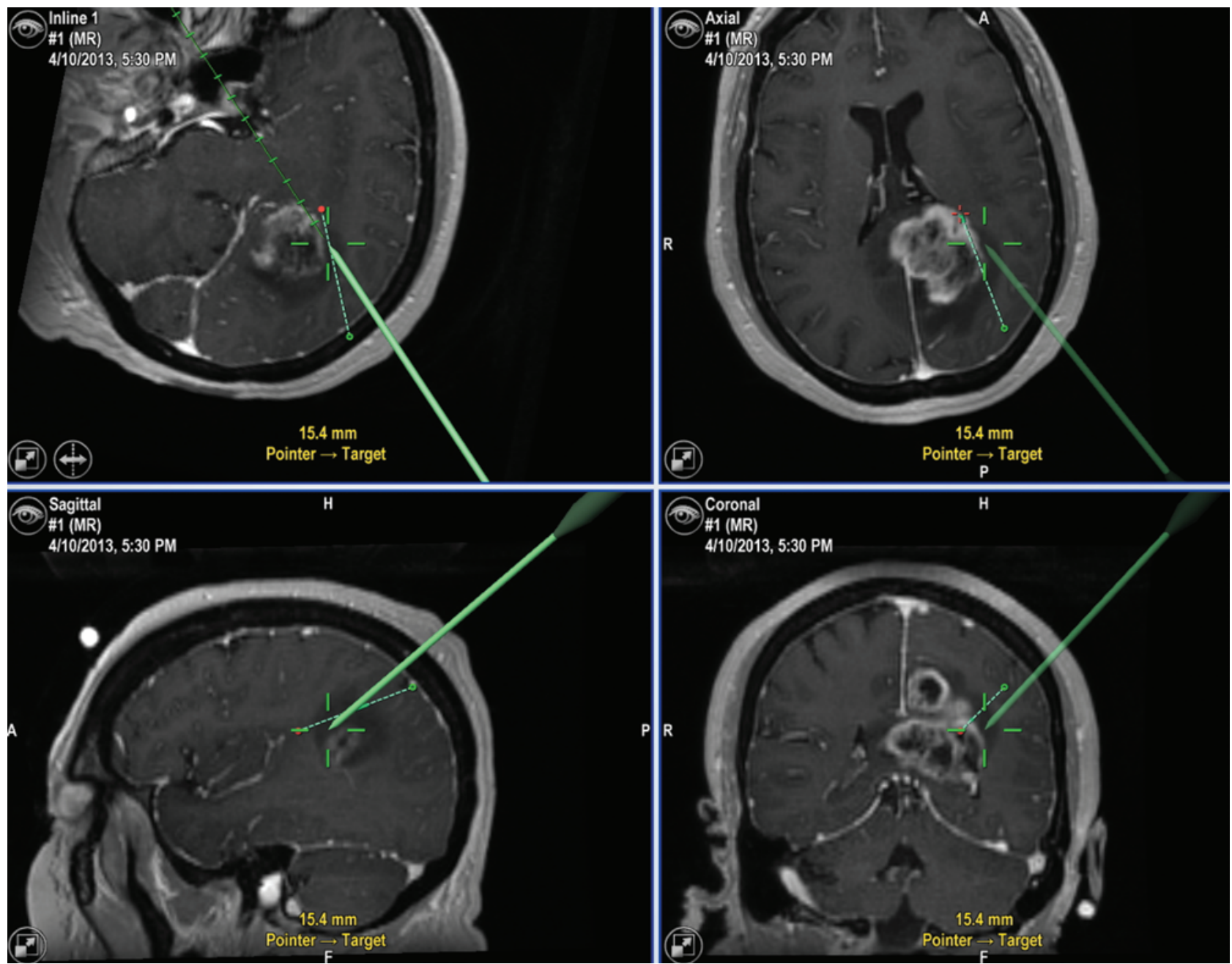

FIG. 6. Intraoperative screenshot of the neuronavigation screen. The point of the initial Indigo Carmine injection is marked with the red dot in the inline view. The crosshairs show the point where the Indigo Carmine injection point was identified intraoperatively. The distance between the 2 points is $15.4 \mathrm{~mm}$, suggesting significant brain shift that can lead either to suboptimal tumor resection or entry into normal brain tissue. Because of the development of the resection cavity and/or intraoperative cerebral edema, this deviation was attributed to brain shift. Figure is available in color online only.

era, a literature review did not yield any reports of adverse events after use of Indigo Carmine in the CNS. LeRoux et al. infused Indigo Carmine intraparenchymally to confirm ultrasonography-guided resections without any adverse events, thus using the injections for a completely different purpose. ${ }^{8}$ Another benefit of Indigo Carmine injections is the low cost and ease of use in centers that do not have access to intraoperative MRI. Only frameless stereotactic equipment is required. The Indigo Carmine dye is available in most urology operating rooms, and the insulin syringe and the 20-gauge spinal needle are commonly available in hospitals. The cost of Indigo Carmine dye is $\$ 9.70$ per ampule (http://store.mcguff.com/products/3431.aspx).

Although an aggressive attempt to achieve gross-total resection might increase the extent of resection, it can unfortunately cause additional neurological deficits in the patient. The current standard of care is the resection of the tumor under frameless stereotactic guidance. This technique is accurate until the point of arachnoid opening; it becomes less accurate after tumor resection has begun.
The reason for this inaccuracy is brain shift, which is the movement of the brain relative to the preoperative imaging study caused by CSF egress, gravity, tissue manipulation, and alterations from peritumoral edema. Nimsky et al. showed that the maximum shifting of the white matter tract ranged from an inward shifting of $8 \mathrm{~mm}$ to an outward shifting of $15 \mathrm{~mm}^{13,14}$ and that the shifts of the cortical surface can reach $23.8 \mathrm{~mm}$ and the shifts of the deep tumor margin range from -7.9 to $+30.9 \mathrm{~mm} .{ }^{12}$ Intraoperative ultrasonography has been introduced to obtain real-time intraoperative images. However, the obtained images cannot reliably differentiate between tumor and artifact or between tumor and normal brain. ${ }^{6}$ As a result, the reliability of intraoperative ultrasonography has been questioned. $3,17,22$

Intraoperative MRI is clearly the most reliable method for determining tumor margins during surgery. However, the equipment is expensive (approximately $\$ 3$ million) ${ }^{20}$ and therefore is only available in few institutions. Moreover, intraoperative MRI requires specialized equipment 
and room shielding, and it introduces certain limitations for the surgeon because some surgical instruments or equipment are not available in MRI-compatible versions. Likewise, the signals from intraoperative MRI can be difficult to interpret because bleeding into the resection cavity and trauma-related artifacts can both obscure and mimic residual tumor. Intravenous contrast enhancement can be used for intraoperative MRI, but its leakage into the peritumoral tissue renders the interpretation of delayed, repeat imaging challenging. ${ }^{5}$

Low-field intraoperative MRI systems are compact and pose fewer limitations in terms of cost and operating room integration, but they have inherent limitations such as increased acquisition time and decreased spatial and temporal resolution. In addition, intraoperative MRI findings might be inconsistent with postoperative high-field imaging findings. ${ }^{19} \mathrm{On}$ the basis of the proposed technique, certain critical locations (that have been identified by specialized MRI sequences) can be marked with the Indigo Carmine dye at the beginning of the operation, and regardless of the degree of brain shift, the coloring will still mark the original injection point. Another use for Indigo Carmine would be marking metabolically active tumor areas on the basis of MR spectroscopy and nuclear medicine study findings and ensuring their resection. Coupled with intraoperative cortical and deep white matter brain mapping, Indigo Carmine injections could be used to identify proximity to white matter tracts preoperatively determined by diffusion tensor imaging.

Recently, to address the need for better intraoperative visualization of the tumor, 5-aminolevulinic acid (5-ALA) was introduced..$^{21}$ 5-ALA produces fluorescence in highgrade neoplasms and facilitates tumor resection and identification of small islands of residual tumor. However, unclear vague fluorescence is a subjective determination, and the correlation of low levels of fluorescence with clear tumor infiltration is debatable. Moreover, fluorescence is not reliably exhibited by nonenhancing tumors, such as most low-grade gliomas. ${ }^{4}$ Although use of intraoperative confocal microscopy might extend the indications of 5-ALA to low-grade tumors, ${ }^{18}$ use of 5-ALA in the United States is not approved by the FDA. Of note, 5-ALA provides selective fluorescence of neoplastic tissue through mitochondrial metabolism of protoporphyrin, whereas Indigo Carmine just stains the tissue where it is injected, which is based on the tumor margin as represented on MR images.

Further studies should delineate the diffusion properties of different tumor pathologies and further characterize the safety profile of the technique. A Phase III study, in which patients will be randomly assigned to receive standardized injections or no injections and the extent of resection is an end point, is planned. In theory, the technique can prove helpful for the following: tumors with optical properties similar to normal brain, tumors with specific morphological characteristics (e.g., fingerlike projections) for which residual postoperative tumor is probable, large tumors for which resection would create a big resection cavity and therefore significant brain shift, and conditions with preexisting or expected intraoperative brain edema. Children were not enrolled in this study, and this age group is a significant field for expanding the current study, especially given the fact that implementing preoperative or intraoperative brain mapping techniques in children is challenging. Last, a controlled study or a comparison of the method with alternative techniques could investigate its comparative merits.

\section{Conclusions}

Stereotactic injection of Indigo Carmine dye can be used to mark the margins of an intraaxial tumor and can potentially help the surgeon increase resection to reach the margins and can act as a signpost for when to stop to avoid a neurological deficit. Because the technique is very easy to implement and the equipment is readily available in most modern operating rooms, the generalizability of the method is facile.

\section{Acknowledgments}

We thank the FDA for insightful review comments during the IND process. We thank Akorn, Inc., for the prompt supply of necessary documentation for the IND process. We appreciate the coordination of the study by Tamika Wong, MPH.

\section{References}

1. Bohman LE, Gallardo J, Hankinson TC, Waziri AE, Mandigo CE, McKhann GM II, et al: The survival impact of postoperative infection in patients with glioblastoma multiforme. Neurosurgery 64:828-835, 2009

2. De Bonis P, Albanese A, Lofrese G, de Waure C, Mangiola A, Pettorini BL, et al: Postoperative infection may influence survival in patients with glioblastoma: simply a myth? Neurosurgery 69:864-868-869, 2011

3. Erdoğan N, Tucer B, Mavili E, Menkü A, Kurtsoy A: Ultrasound guidance in intracranial tumor resection: correlation with postoperative magnetic resonance findings. Acta Radiol 46:743-749, 2005

4. Floeth FW, Sabel M, Ewelt C, Stummer W, Felsberg J, Reifenberger G, et al: Comparison of (18)F-FET PET and 5-ALA fluorescence in cerebral gliomas. Eur J Nucl Med Mol Imaging 38:731-741, 2011

5. Foroglou N, Black PM: Mid-field suite design, in Hall WA, Nimsky C, Truwit CL (eds): Intraoperative MRI-Guided Neurosurgery. New York: Thieme, 2011, pp 12-17

6. Gerganov VM, Samii A, Giordano M, Samii M, Fahlbusch R: Two-dimensional high-end ultrasound imaging compared to intraoperative MRI during resection of low-grade gliomas. J Clin Neurosci 18:669-673, 2011

7. Kajiwara K, Yoshikawa K, Ideguchi M, Nomura S, Fujisawa $\mathrm{H}$, Akimura T, et al: Navigation-guided fence-post tube technique for resection of a brain tumor: technical note. Minim Invasive Neurosurg 53:86-90, 2010

8. LeRoux PD, Berger MS, Ojemann GA, Wang K, Mack LA: Correlation of intraoperative ultrasound tumor volumes and margins with preoperative computerized tomography scans. An intraoperative method to enhance tumor resection. J Neurosurg 71 (5 Pt 1):691-698, 1989

9. McGirt MJ, Chaichana KL, Attenello FJ, Weingart JD, Than $\mathrm{K}$, Burger PC, et al: Extent of surgical resection is independently associated with survival in patients with hemispheric infiltrating low-grade gliomas. Neurosurgery 63:700-708, 2008

10. McGirt MJ, Chaichana KL, Gathinji M, Attenello FJ, Than K, Olivi A, et al: Independent association of extent of resection with survival in patients with malignant brain astrocytoma. Clinical article. J Neurosurg 110:156-162, 2009 
11. Mixter WJ: Ventriculostomy and puncture of the floor of the third ventricle. Boston Med Surg J 188:277-278, 1923

12. Nimsky C, Ganslandt O, Cerny S, Hastreiter P, Greiner G, Fahlbusch R: Quantification of, visualization of, and compensation for brain shift using intraoperative magnetic resonance imaging. Neurosurgery 47:1070-1080, 2000

13. Nimsky C, Ganslandt O, Hastreiter P, Wang R, Benner T, Sorensen AG, et al: Intraoperative diffusion-tensor MR imaging: shifting of white matter tracts during neurosurgical procedures - initial experience. Radiology 234:218-225, 2005

14. Nimsky C, Ganslandt O, Hastreiter P, Wang R, Benner T, Sorensen AG, et al: Preoperative and intraoperative diffusion tensor imaging-based fiber tracking in glioma surgery. Neurosurgery 56:130-138, 2005

15. Orringer DA, Golby A, Jolesz F: Neuronavigation in the surgical management of brain tumors: current and future trends. Expert Rev Med Devices 9:491-500, 2012

16. Rachel RA: Surgical treatment of hydrocephalus: a historical perspective. Pediatr Neurosurg 30:296-304, 1999

17. Rygh OM, Selbekk T, Torp SH, Lydersen S, Hernes TA, Unsgaard G: Comparison of navigated 3D ultrasound findings with histopathology in subsequent phases of glioblastoma resection. Acta Neurochir (Wien) 150:1033-1042, 2008

18. Sanai N, Snyder LA, Honea NJ, Coons SW, Eschbacher JM, Smith KA, et al: Intraoperative confocal microscopy in the visualization of 5-aminolevulinic acid fluorescence in lowgrade gliomas. Clinical article. J Neurosurg 115:740-748, 2011

19. Senft C, Seifert V, Hermann E, Franz K, Gasser T: Usefulness of intraoperative ultra low-field magnetic resonance imaging in glioma surgery. Neurosurgery 63 (4 Suppl 2):257-267, 2008

20. Sherman JH, Hoes K, Marcus J, Komotar RJ, Brennan CW, Gutin PH: Neurosurgery for brain tumors: update on recent technical advances. Curr Neurol Neurosci Rep 11:313-319, 2011

21. Stummer W, Pichlmeier U, Meinel T, Wiestler OD, Zanella F,
Reulen HJ: Fluorescence-guided surgery with 5-aminolevulinic acid for resection of malignant glioma: a randomised controlled multicentre phase III trial. Lancet Oncol 7:392401, 2006

22. Tronnier VM, Bonsanto MM, Staubert A, Knauth M, Kunze S, Wirtz CR: Comparison of intraoperative MR imaging and 3D-navigated ultrasonography in the detection and resection control of lesions. Neurosurg Focus 10(2):E3, 2001

\section{Author Contributions}

Conception and design: Schwartz, Margetis. Acquisition of data: Schwartz, Margetis, Rajappa, Tsiouris. Analysis and interpretation of data: Schwartz, Margetis, Rajappa, Tsiouris. Drafting the article: Schwartz, Margetis. Critically revising the article: all authors. Reviewed submitted version of manuscript: all authors. Approved the final version of the manuscript on behalf of all authors: Schwartz. Statistical analysis: Margetis. Administrative/ technical/material support: Study supervision: Schwartz.

\section{Supplemental Information Videos}

Video 1, Media Player. http://mfile.akamai.com/21490/wmv/ digitalwbc.download .akamai.com/21492/wm.digitalsource-naregional/jns14-113_video_1.asx.

Video 1, Quicktime. http://mfile.akamai.com/21488/mov/ digitalwbc.download.akamai.com/21492/qt.digitalsource-global/ jns14-113_video_1.mov.

\section{Correspondence}

Theodore H. Schwartz, Weill Cornell Medical College, 525 E. 68th St., New York, NY 10065. email: schwarh@med.cornell. edu. 